IX. Ueber die Richtung der Schwingungen des

Lichtäthers im geradlinig polarisirten Lichte;

von $W$. Haidinger.

(Mlitgetheilt vom Hrn. Verf. aus den Sitzungsberichten d. Viener Akadernie 1852 Januar.)

1. Physikalischer Beweis für den Satz, dafs die Schwingungen des Lichtäthers im geradinig polarisirten

Lichte auf der Polarisationsebene senkecht stattinden.

1. Beobachlung. Man betrachte einen dichromatischen einaxigen Krystall in einer auf der Krystallaxe senkrecht stehenden Richtung.

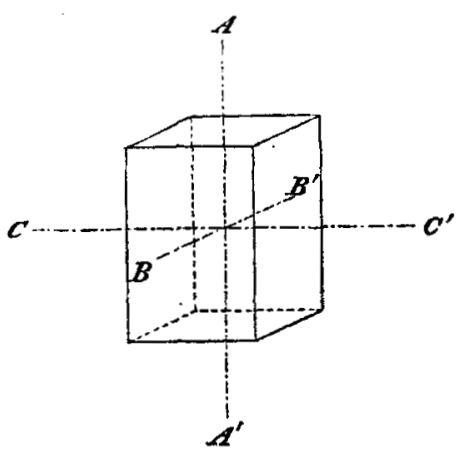

Die Linie $A A^{\prime}$ in beistehender Figur ist die Axe des Krystalls, $B B^{\prime}$ die Längsrichtung, in welcher das Auge den Krystall betrachtet, $C^{\prime \prime}$ die Querrichtung, senkreclt auf den beiden andern.

Man betrachte den Krystall erleuchtet von gewöhnlichem Lichte, und untersuche die Erscheinung desselben durch die dichroskopische Lupe in allen Azimuten senkrecht auf der Axe. Da von den zwei Bildern der dichroskopischen Lupe das eine ordinär, in der Richtung beider Bilder, das andere extraordinär, senkrecht auf dieser Richtung polarisirt ist, so erscheinen sie auch von den zwei verschiedenen Farbentönen des dichromatischen Krystalls verschieden gefärbt.

Wie immer man auch bei gleicher Stellung des Auges und der dichroskopischen Lupe den Krystall un die Axe $A A^{\prime}$ herumdrehen mag, immer bleibt die Erscheinung gleich; 


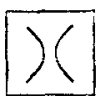

$O$ das obere Bild $O$, in beistehender Figur, in der Richtung der Axe polarisirt und von der einen Durchsichtigkeits-Farbe $A$, das untere Bild $E$ $\asymp$ $E$ senkrecht auf der Axe polarisirt und von der andern Durchsichtigkeits - Farbe $B$ des Krystalls.

Betrachtet man den Krystall in der Richtung der Axe, so erscheinen beide Bilder der dichroskopischen Lupe von gleicher Farbe, und zwar von der Farbe $A$, wie das ordiuäre Bild der Beobachtungen senkrecht auf der Axe.

2. Lehrsatz. Die Schwingungen des Lichtäthers stehen senkrecht auf der Polarisationsebene.

3. Beweis. Im gewöhnlichen Lichte stehen die Schwingungsrichtungen des Lichtäthers in allen Azimuten senkrecht auf der Fortpflanzungsrichtung. In geradlinig polarisirten Lichte liegen alle Schwingungsrichtungen in einer einzigen Ebene.

Bei jeder Beobachtung senkrecht auf der Axe erscheinen zwei Lichtstrahlen, die senkrecht gegen einander polarisirt sind. Es wird also für jede einzelne Beobachtung zwei Schwingungsebenen geben. Jede derselben ist bei dem durchaus homogenen Krystall durch eine eigenthümliche Farbe ausgezeichnet. Für die Summe der Bcobachtungen rund um die Axe herum giebt es eine Schwingungsrichtung in der Richtung der Axe, und eine unendliche Anzahl von Schwingungsrichtungen, die sämmtlich in der senkrecht auf der Axe stehenden Ebene liegen.

In der Richtung der Axe erscheint nur eine Farbe, aber man beobachtet sie in allen Azimuten. Zu ihr gehören offenbar alle senkrecht auf der Axe stehenden Schwingungen, welche in der gleichen Farbe auch bei den Beobachtungen senkrecht auf der Axe wahrgenommen werden.

Die andere Farbe beobachtet man in der Richtung der Axe gar nicht, wohl aber in allen Azimuten rund un dieselbe. Für sie bleibt die einzelne in der Richtung der Axe stattfindende Schwingungsrichtung übrig. Die Beobachtung zeigt, dafs diese Farbe senkrecht auf der Axe po- 
larisirt ist. Sie wird aber durch die Schwingungen in der Richtung der Axe bedingt, also stehen anch die Schwingungen senkrecht auf der Polarisationsebene; was zu erweisen war.

Man könnte die Beweisführung auch so ausdrücken: Die drei senkrecht auf einander stehenden Linien seyen $\boldsymbol{A} \boldsymbol{A}^{\prime}$ die $A x e, B B^{\prime}$ die Läugsdiagonale, $C C^{\prime}$ die Querdiagonale. Für diejenige Farbe, welche nur um $B C B^{\prime} C^{\prime}$ herum, aber nicht in der Richtung der Axe, beobachtet wird, sind aus dem Punkte $B$ betrachtet die Schwingungen parallel $A A^{\prime}$ oder parallel $C C^{\prime}$, gewifs nicht parallel $B B^{\prime}$, denn diese wären longitudinal. Aus dem Punkte $C$ betrachtet wären sie eben so gewifs parallel $A A^{\prime}$ oder parallel $B B^{\prime}$, gewifs nicht parallel $C C^{\prime}$, deun diese wären wieder longitudinal. $\mathrm{Zu}$ einer Farbe gehört aber nur eine Richtung von Schwingungen, $B B^{\prime}$ und $C C^{\prime}$ sind beide ausgeschlossen, alser $A A^{\prime}$ bleibt als die allein mögliche Schwingungsrichtung übrig. Sie steht senkrecht auf der Ebene $B C B^{\prime} C^{\prime}$. Aber diese ist senkrecht auf der Axe polarisirt, also stehen die Schwingungen auf der Polarisationsebene senkrecht.

Eine kleine Kugel von Turmalin, an welcher der ordinäre Strabl absorbirt ist und nur der extraordinäre noch Farbe und Durchsichtigkeit zeigt, kann als genaues Modell bei der gegenwärtigen Betrachtung dienen. Für die hellere Farbe existirt augensclicinlich keine auf der Axe senkrecht stehende Schwingung,' sonst würde die Farbe auch in der Richtung der $\Lambda x e$, und nicht blofs senkrecht darauf sichtbar seyn. In gewöhnlichen Lichte erscheint hier in einem besonderen Falle, was bei den dichromatischen Krystallen im polarisirten Lichte immer beobachtet wird.

4. Anwendung. Da nun die verticalen der Axe parallelen Schwingungen zu der extraordinären Polarisationsebene gehören, so bleiben in jeder einzelnen Beobachtung für die ordinäre Polarisationsebene die horizontalen Schwingungen übrig, welche auf ihr senkrecht stehen. Man mufs aber daun die gleiche Farbe auch über die Endfläche senk- 
recht auf der Axe hinüber stets in der Beobachtung haben, und diefs ist wirklich der Fall; man sieht stets zwei gleichgefärbte Bilder, deren Polarisationsebenen senkrecht auf einander stehen. Dadurch wird endlich auch für die dritte, senkrecht auf den beiden vorhergehenden stehende Polarisationsebene, wenn es ja erforderlich wäre, bewiesen, dals auch auf ihr die einzige noch übrig bleibende Schwingungsrichtung senkrecht steht.

5. Trichromatische Krystalle. Der gleiche Beweis wie oben kann auch mit den Erscheinungen an einem zweiaxigen Krystalle geführt werden. Er stimmt in sowcit mit dem einaxigen überein, dafs die azimutal senkrecht gegen einander stehenden Beobachtungen senkrecht auf der Axe jedesmal zwei senkrecht auf einander polarisirte Lichtstrahlen enthalten, welche durch die dichroskopische Lupe getrennt werden können, ferner noch darin, dafs der extraordinäre Strahl rund um die Axe herum die gleiche Farbe hat. Die ordinär polarisirten Strahlen haben aber nicht die gleichen Farben, sondern zeigen zweierlei Töne, deren Maximum-Unterschiede senkrecht gegen einander gestellt sind. Die extraordinäre Farbe $B$ bleibt, die Farbe $A$ aber theilt sich in zweierlei Töne, deren einen man ferner $A$ nennen kann, während der andere $C$ heilsen mag. Die beiden Töne $A$ und $C$ treffen mit senkrecht gegen einauder stehender Polarisation auf der Basis, oder der senkrecht auf der Axe stehenden Ebene zusammen. Jede derselben ist um das ganze Azimut, die eine um die Queraxe, die andere un die Längsaxe sichtbar. Die Schwingungen, den Farben entsprechend, stehen überall senkrecht auf den Polarisationsebeuen.

\section{I. Bemerkungen.}

Ich glaube, dafs es ummöglich ist, einen Beweis für den in Rede stehenden Satz mit gröfserer Anschaulichkeit, Sicherheit und Einfachheit aufufinden.

$\mathrm{Ob}$ er noch wünschenswerth war? Ich glaube auch hier ein Ja aussprechen zu könuen. Zwar verschswindet 
die Ansicht, dafs die Schwingungen in der Polarisationsebene liegen, immer mehr, aber doch ist der Fortgang der Ausbreitung der entgegengesetzten Ansicht, dafs sie näılich senkrecht auf der Polarisationsebene stehen, die gewifs die einzig richtige seyn kaun, noch immer von einigen Seiten beanstandet. "Die Erscheinung der Polarisationsbüschel, "sagt Moigno, "nufs natürlich die so zarte Frage der Richtung der Schwingungen der Aether-Molecule in Bezug auf die Polarisationsrichtung wicder auf den Platz bringen, eine Frage, auf die wir schon so oft zurück gekommen sind, und die, um aus den entgegengesetzten Behauptungen zu schliefsen, welche täglich vorgebracht werden, noch vollständig unentschieden wäre').

Bekanntlich war es Fresnel, der zuerst von der Voraussetzung ausging, dafs bei dem polarisirten Lichte die Schwingungen senkrecht auf der Polarisationsebene stehen. Cauch y war der erste, der, den Ansichten Fresnel's entgegen, annahm, die Schwingungen fäuden statt in der Ebene der Polarisation selbst. Doch hatte er, wie sich Mo ign o ausdrückt, "den glorreichen Muth, seine Ansicht zurückzuziehen, um wieder auf Fresnel's Theorie zu kommen. "

Hr. Abbé Moigno giebt an mehreren Stellen seines Répertoire die verschiedenen in Bezug auf den Gegenstand vertheidigten Ausichten. Er selbst nimmt uubedingt die Ansichten Fresnel's und die damit übereinstimmenden Cauchy's an ${ }^{2}$ ). In der Auseinandersetzung der Metho-

1) La présence des houppes dans la lamière polarisée soulète naturellement la question si délicate de la direction des vibrations des molécules éthérées par rapport au plan de polarisation, question sur luquelle nous sommes dejà revenus lant de fois, et qui, à en juger par les assertions contraires qui se produisent chaque jour, serait encore complètement indécise. Maigno, Répertoire d'Optique moderne, $I F$, p. 1365.

2) J'ai dit avec détail dans la première parlic de ce travail comment $\boldsymbol{M}$. Cauchy arait pit étublir rigoureusement les formules qui donnent les intensités des rayons réféchis et réfractés; je suis toujours contuincu que la marche suicie par billustre góo- 
den von Mac Cullagh und Neumann ist unter mehreren Grundsätzen, welche als Axiome oder Hypothesen ohne Beweis angenommen werden, auch der Folgende: Im polarisirten Lichte siud die Schwingungen der Polarisationsebene parallel ${ }^{1}$ ). Kürzlich noch hat Hr. Babinet in einer Mittheilung an die Academie in Paris behauptet, dafs die Schwingungen in der Polarisationsebene selbst stattfinden ${ }^{2}$ ). Er koumt freilich bei Moigno zicmlich übel weg. Wichtig ist es aber insbesondere für den Zweck der gegenwärtigen Mittheilung, dafs in der Antwort Cauchy's auf Babinet's Behauptung der vollständige Beweis desselben enthalten ist, den ich hier gerne zum Vergleiche mit dem oben gegebenen beifügc. Cauchy nennt ihn "einen in wenigen Worten gegebenen so einfachen Beweis, dafs er, wie er hofft, genizgen wird, alle Ungewifsheit zu zerstreuen ${ }^{3}$ ).

"Niemand bestreitet heute mehr die Schlüsse, zu welchen ich in den der Academie in den Jahren 1829 und 183! vorgelegten Abhandlungen gelangt bin, nämlich dafs ein elastisches Mittel, wenn die Elasticität in allen Richtungen gleich bleibt, fähig ist, zweierlei Schwingungs-Syteme cbener Wellen fortzupflanzen. Die Schwingungen können cntweder tranversal seyn, d. h. den Ebenen der Wellen parallel, oder nicht transversal. Es ist für den Augenblick gleichgültig, ob die nicht transversalen Schwingungen longitudinal, d. h. senkrecht auf den Ebenen der Wellen, sind oder nicht; dafs sie in gröfseren oder geringeren

mètre est seule logique, seule uraiment démonstrative. Ripertoire t. I, p. 111.

1) Répertoire, t. I, p. 112.

2) "De ces deux expériences réunies, je déduis, que les cibrations lumineuses d'un rayon polurisé s'éxecutent dans le plan méme de polurisation;" Babinet in der Ablandlung:" Sur le sens de vilrations dans les rayons palarisés. Moigno, Répertoire, t. IF, p. 1366.

3) J'en donnerai en peu de mots une démonstration tellement simple, qu'elle suffira, je lespère, puur dissiper toute incertitude.

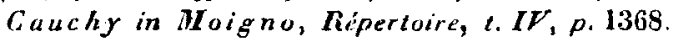


Entfernungen von den zurückstrahlenden Oberflächen mehr oder weniger bemerkbar, oder ob sie selbst, was man in der Theorie des Lichts zum Verschwinden abnehmende Lichstrahlen nenut, hervorbringen. Gewifs ist in allen Fällen, dafs wenn ebene Wellen durch die TrennungsOberfläche zweier Mittel zurückgeworfen oder gebrochen werden, dic nicht transversalen Schwingungen in Ebenen stattfiuden, welche senkrecht auf den Durchschnitten (traces) der Ebenen der Wellen auf der Oberfläche selbst stehen. “

„Dieses rorausgesetzt, falle ein einfacher Lichtstrahl auf die Trennungs-Oberfläche zwischen zwei isophanen Mitteln. Man setze ferner voraus, dafs in diesem Lichtstrahl die Schwingungen der Aethertheilchen nicht nur transversal, sondern auch noch der besagten Oberfläche parallel seyn, folglich auch parallel den Durchschnitten (traces) der Ebenen der Wellen mit derselben Oberfläche. Diese Transversal-Schwingungen werden wohl, in den zurückgeworfenen oder gebrochenen Wellen, zu anderen transversaleu Schwingungen Anlafs geben können, deren Richtungen ihren cigenen Richtungen parallel sind, aber augenscheinlich niemals zu nicht transversalen Schwingungen, welche in Ebenen stattfänden, die jene Richtungen rechtwinklich schneiden würden. Daher werden nicht transversale Schwingungen im Lichtäther niemals durch die Zurückstrahlung und die Brechung hervorgebracht werden können, wenu die Schwingungen der Molecule der Zurückstrahlung:sfläche parallel sind. “

"Nimmt man diesen Grundsatz an, der, wie mir scheint, unbestreitbar ist, so findet sich die Frage, ob die Polarisationsebene senkrecht auf der Richtung der Aetherschwingungen sey oder nicht, vollkommen aufgelöst. Wirklich, wenn dic Schwingungen im einfallenden Strable der Zurückstrahlungsfläche parallel sind, dann werden nach den vorhergehenden Betrachtungen die einzigen Wellen, welche in dem einen Mittel durch Zurückstrahlung, in dem andern durch Brechung hervorgebracht werden, Wellen mit Transversal-Schwingungen seyn, und die Richtung dieser Schwin- 
gungen ist die gleiche in dem einfallenden, in dem zurückgeworfenen und in den gebrochenen Strahl. Wenn ferner der zurückgeworfene Strahl merklich verschwinden könnte, wenigstens unter einem gewissen Winkel, dann dürtte, entsprechend dem Grundsatze der Continuirlichkeit in der Bewegung des Aethers, der gebrochene Strall weder durch seine Richtung noch durch seine Beschaffenlueit von dem einfallenden Strahle verschieden seyn. Da indessen diese Bedingung niemals erfuillt wird, wenn der Brechungsexponent von der Einheit verschieden ist, so mufs man schliefsen, dafs jeder einfallende Strahl, in welchem die Schwingungen der Zurückstrahlungsebene parallel sind, und daher auch senkrecht auf der Einfallsebene stehen, in die Zahl derjenigen gehört, welche die Zurückstrahlung nicht verschwinden machen kann. Aber ein solcher Strahl ist es gerade, den man einen in der Einfallsebene polarisirten Strahl nenut. Also steht die Polarisationsebene senkrecht auf der Richtung der Schwingungen der Lichtäther-Molecule ').

1) Personne, anjuurd'bui, ne conteste plus les conchesions auxquelles je suis purvenu dans les mérmaires présentés ì l'Académie en 1829 et 1830, savoir, qu'un milieu ćlustique, quand l'élusticité reste la méme en tous sens, est cupable de propager deux espèces de mowverments sibratoires d̀ ondes planes. Les vibrations peusent étre, ou transsersales, c'est-d-dire paralleles aux plans dis ondes, ou non transuersales. Que les vibrations non transcersales soient ou ne soient pas tongitudinales, c'est-à-dire perpendiculuires uux plans des ondes; qu'elles soient plus ou moins sensibles ì des distances plus ou moins grandes des surfaces réflichissantes, ou puissent méme constiluer ce qu'on appelle, dans la théoric de la lumière, des rayons évanescents, peu importe pour le mornent. Il est certain, dans tous les cas, que si des undes planes sont réfléclies ou réfractées par la surface de síparation de deux milieux. Les vibrations non transwersales sexéculent dans des plans perpendiculaires anx traces des plans des ondes sur lit surface elle-méme.

Cela posé, concerons qu'un rayon lumine'ux siniple tombe sur Ia surface qui sipare l'un de l'antre derax milicux isophanes. Supposons, d'ailleurs, que, daus ce rayon, lis sibrations des molrcules élhérés soient, nun-senlement transiersales, mais encore 
Gewifs ist diese Beweisführung viel umständlicher als die oben angegebene, und auch wohl weniger klar. $\mathrm{Zu}$ dem kommt in derselben eine Stelle vor, die einer Erklärung bedürfte. Es heifst nämlich: "Die Richtung dieser (der transversalen, der Zurückstrahlungsfläche parallelen Schwingungen) ist die gleiche in dem einfallenden, in dem zurückgeworfenen und in dem gebrochenen Strahl. " Es ist freilich wahr und wird durch die Beobachtung bestä-

parallèles à lit surface dont it s'agit, et par conséquent aux trace's des plans des ondes sur celle méme surface: ces vibrations transversales pourront bien donner naissance, dans les ondes réftéchies ou réfractées, à d'autres vibrations transwersales, dont les dircctions soient paralleles à leurs directions propres, mais jamais, évidemment, ̀̀ des vibrations non transwersales compriscs dans des plans qui couperaient ces directions ì angles droits. En cunséquence, les vibrations non transwersales ne pourront jamais étre produites, dans le fluide éthéré, par la réflexion et la réfraction d'un rayon lumineux, lorsque les vibrations des molécules seront parallèles ì la surface réfléchissante.

Ce principe, qu'il me scmble impossible de contester. étant admis, la question de savoir si le plan de politrisation d'un rayon lumineux est ou n'est pas perpendiculaire aux direclions des wibrations d'éther se trouve complètement résolue. En effet, si, dans le rayon incident, les vibrations sont parallèles à la surface réfléchissante, alors, d'après ce qu'on vient de dire, les seules ondes produites dans le premier nilieu par lie réflexion, et dans le second milieu par la réfraction, seront des ondes à vibrations transversales, les directions de ces vibrations étant les mémes dans les rayons incidents, réfléchis et réfractés. Si d'ailleurs le rayon réfléchi poucait s'évanouir sensilllement, au moins sous un certain angle, alors, en vertu du principe de la continuité du mouvement dans l'éther, le rayon réfracté ne deurait différer, ni par st direction, ni par sa nature, du rayon incident. Or, cette condition n'étant jamais remplie, quand l'indice de réfraction diffère de l'unité, on doit en conclure que tout rayon incident dans lequel les vibrations sont paralleles à la surface réfléchissante, et par conséquent perpendiculuires au plan d'incidence, est du numbre de ceux que la réflexion ne peut faire disparaitre. Mrais un tel rayon est précisément ce qu'on appelle in rayon polarisé dans le plan d'incidence. Douc le plan de polurisation est perpendiculaire aux directions des vibrations moléculaires. Cauchy in Moigno, Répertuire etc., t. IV, p. 1368. 
tigt, dafs ein bereits in der Einfallsebene polarisirter StrahI einen in der Einfallsebene polarisirten zurückgeworfenen, und einen in der Einfallsebene polarisirten gebrochenen Strahl giebt; in diesem Falle sind in der That die Schwingungen gleich. Anders ist es, wenn der einfallende Strabl in zwei senkrecht auf einander stehenden Richtungen polarisirtes, oder auch wenn er ordinäres, in allen Richtungen polarisirtes Licht ist. Dann ist der zurückgeworfene Strahl zwar in der Einfallsebene, und zwar unter dem Polarisationswinkel ziemlich vollständig, polarisirt, aber von dem gebrochenen oft nur theilweise polarisirten Strahle ist der polarisirte Antheil bekanntlich nicht in der Einfallsebene, sondern senkrecht anf denselben polarisirt. Nar durch einen Intensitäts - Teberschufs kann bei schon vor dem Einfall in der Einfallsebene polarisirtem Lichte auch nach der Brechung noch dieselbe Polarisationsrichtung vorwalten. Sie verschwindet übrigens nach mehrmaligem Durchgange durch unter dem Polarisationswinkel geneigte Glasplatten ginzzlich, ohne dafs die Polarisation senkrecht auf der Einfallscbene übrig bleibt, wie bei ordinärem Lichte, weil schon kein in dieser letztern Richtung polarisirter Bestandtheil vorausgesetzt wurde.

Man kann die zur Prüfung erforderlichen Beobachtungen sehr leicht mit einer dichroskopischen Lupe, welche die zwei gegen einander senkrecht polarisirten Strahlen im Gegensatze zeigt, und einer Auzahl unter dem Polarisationswinkel zwischen dieselbe und das Auge gebrachter Glasplatten vornehmen.

Mit dem Ausrufe: In cauda venenum, führt freilich nun auch Hr. Abbé Moigno an, dafs Hr. Babinet die gauze Auseinandersetzung nicht für einen Beweis gelteu lassen will. Zugleich bestreitet er diese Nichtzustimmung, und erklärt sich für Hrn. Ca uch y's gegenwärtige Ansicht, aber man wird es bei genauer Prüfung zugeben, kaum mit Worten, die auf irgend eine Beweiskraft Anspruch machen können. Zum Abschlufs heifst es: "Wir haben also von einer Seite Fresuel und Hm. Cauchy, dic grölste 
physikalische und die gröfste mathematische Autorität; von der anderen die HH. Babinet, Mac Cullagh, Neumann und Broch; von der einen Seite cine wundervolle Fruchtbarkeit, fast eine Schöpfung, leichte Erklärungen aller Phänomene, natürliche Auslegung aller Formeln, eine untrügliche Voraussicht einer Menge noch unbekannter Thatsachen u. s. w.; von der andern unfruchtbare Abstractionen, verdrehte und schwerfällige Auslegungen und Bestätigungen u. s. w.; Jeder möge nun selbst wählen! " ').

Die Wahl ist freillch auf das Leichteste vorbereitet, zumal da überhaupt Fresnel's und Cauchy's Ansichten immer allgemeiner angenommen werden, wie denn auch in unserem Kreise die grofsen Mathematiker und Physiker v. Ettingshausen und Petzval sie vertheidigen; aber die Thatsache selbst, dafs die entgegengesetzten Meinungen bestanden und zum Theil noch bestehen, macht gewifs jede Betrachtung von einer neuen Seite wünschenswerth. So sagt unter anderem Hr. Dale: "Hr. Green nimmt als Theil der Grundlage seiner Rechnungen die ursprüngliche Ansicht Fresnel's an - dafs die Schwingungen eines polarisirten Lichtstrahles auf der Polarisationsebene senkrecht stehen; da aber dieser Punkt ein Gegenstand von Streit unter den Mathematikern ist, so habe ich eine Methode ersonnen, wie man unabhängig von aller Theorie, so viel ich glaube, diesen Punkt entscheiden könnte “ ${ }^{2}$ ).

1) Nous atons donc d'un côté Fresnel et $\boldsymbol{M}$. Cauchy, la plus grande autorité physique, et la plus grante antorité mathémaligat; d: lautre, MIM. Babinet, Mac-Cullagh, Neumann et Broch; d'un co's une fécondité prodigieuse, presque une création, des explications faciles de tous les plínomènes, des interprélations nalurclles de toutes les formules, la prévision infaillible d'une foule de faits inconnus, etc.; de l'autre, des abstractions stériles, des interprétatiuns et confirmations détournées et pénibles, etc., etc.; que chacun choisisse! Moigno Répertoire, T. $1 \mathrm{~V}, \mathrm{p} .1370$.

2) Mr. Green adopts, as part of the basis of his calculation, the original siew of Fresnel, - that the vibrations of a polurized ray are perpendicular to the plane of polarization; but as this 
Sie bestand darin, müglicherweise Veränderungen in den Interferenzen polarisirter Lichtstrahlen hervorzubrin. gen, wenn man sie der Länge nach durch einen in einer Richtung senkrecht auf seiner Länge vermittelst seitlich angewandter Schrauben-Pressung gebogenen 4-5 Zoll langen, und $\frac{1}{2}$ Zoll breiten Glasstab hindurchgehen liefsc. Sir John Herschel bezweifelte jedoch den Frfolg der vorgeschlagenen Versuche ').

Die Frage der Schwingungen ist, wie Hr. Moigno oben bewerkte, allerdings sehr innig mit der Erscheinung der Polarisationsbüschel verbunden. Als ich die erste Mittheilung über diese machte, durfte auch ich die Frage nicht unberührt lassen, die mir so fremd war und in der sich die höchsten wissenschaftlichen Autoritäten für oder wider ausgesprochen hatten. Mein Votum mufste anmafsend oder geringfügig erscheinen. Ich inufs heute den Forschern in dieser Abtheilung unserer Kenntnisse in der That dafür dankbar seyn, dafs die Ansicht, wie ich sie eben aussprechen zu müssen glaubte, schonend erwähnt wurde und für sich dahin gestellt blieb. Es schien mir zuerst ${ }^{2}$ ), die Schwingungen müfsten in der Längen-Richtung der Polarisationsbüschel gehen, die gelbe Farbe mülste gewissermalsen als eine Summirung der einzelnen Eindrücke erscheinen, und unter dieser Voraussetzung hätten die Schwingungen in der Polarisationsebene, nicht senkrecht darauf stattgefunden. Hr. Abbé Moigno hat trefflich und naturgemäls angemerkt, dafs es ihm "unmöglich scheine, nicht zuzugeben, dafs die Molecularschwingungen senkrecht auf der Polarisationsebene stehen, wie es Fresnel wollte, und wie es heute Hr. Cauchy gegen die HH. Neumann, Mac Cullagh, Broch, Babinet u. s. w.

point is a matter of dispute among mathematicians, I have thought of on experimental method by which this point might, as I think, be decided, independently of all theory. Mr. Dale. On Elliptic Polarization. Report of the sixteenth Meeting of the British Association at Southampton 1846. Notices and Abstracts p. 7.

1) Moigno, Répertoire, t. IV, p. 1391.

2) Poggendorff, Bd. LXIII, S. 29, Moigno, Répertuire, I, p. 344. 
bestätigt, weil das Phänoınen Jer Färbung sich rechts und links von der Polarisationsebene sclbst verbreitet " ${ }^{1}$ ). Ich habe später ${ }^{2}$ ) die Lage der Schwingungselemente gegen die Polarisationsebene nach beiden Hypothesen graphisch dargestellt, doch glaubte ich mich nicht über die Wahrscheinlichkeit der cinen oder der andern aussprechen zu dürfen, was mir den Heroen der optisch-mathematischen Forschungen als Attribut anzugehören schien. Es wäre freilich schon damals nicht ganz entfernt gelegen, die Betrachtung anzustellen, dals wenn entlang dem Durchschnitt der auf der Sehaxe stehenden Ebene mit der Polarisationsebene dic transversalen Schwingungen senkrecht auf der letzteren Ebene stehen, durch das Product eines beliebig auf der Polarisationsebene abgeschnittenen Stückes $A$, mit der senkrecht darauf stehenden Schwingungsamplitude $b$, eine wahre wenn auch ganz schmale Fläche dargestellt wird, die vielfach auf eiuander gelegt cine eben so wirkliche Fläche $A B$ mit linearer Polarisation vorstellen kann. Im Gegentheile stellt das Product von $A$ mit einer Schwingungsamplitude $a= \pm b \vee-1$, immer wieder keine wirkliche wenn auch schmale, sondern nur eine imaginäre Fläche, von dem Gesichtspunkte der Betrachtung also nur eine wahre mathematische Linie vor, deren Breite absolut $=0$ ist, also, selbst vielfach zusammengenommen, nie einc Erscheinung geben hann. In Ermanglung von etwas Besserem wäre auch diese Betrachtung nicht ganz unangemessen gewesen. Man hätte sie auch immerhin als Basis gelten lassen können, um anzunehmen, dafs die Transversal-Schwingungen eines polarisirten Lichtstrahles senkrecht auf der Polarisationsebene stehen. Eine vermehrte Wahrscheinlichkeit

1) Puistue le plénomène de coloration s'étend à droite et ì gruche de ce plan, il the semble impossible de ne pas admettre que les vibrations moléculaires sont perpendiculaires an plan de polarisalion, comme le voulait Fresnel, ct comme l'affirme aujourd'hui M. Cauchy contre MM. Neumann, Mac.Culligh, Broch, Babinet etc. Moigno, Répertoire, t. IV, p. 1330.

2) Poggendorff, Bd. LXVIII, S. 73 und 305. Moigno, Répertoire, t. $I F, p .1346$. 
erhält dic Betrachtungsart freilich erst jetzt, wo man nach dem durch die pleochromatischen Krystalle gegebenen Beweise zu sagen berechtigt ist, dafs man wei/s, die Schwingungen stehen senkrecht auf der Polarisationsebene.

Man kanu nun mit vollem Rechte sagen, die Erscheinungen der Farbenvertheilung in den dichronatischen und trichromatischen Krystallen bilden eine glänzende Bestätigung von Fresnel's und Cauchy's Theorie, dafs die Schwingungsrichtungen des Lichtäthers senkrecht auf den Polarisationsebenen stehen.

Der im Gegenwärtigen gegebene Beweis dürfte un so klarer und überzeugender erscheinen, als er von den in Rede stehenden Theorien selbst unabhängig ist. Der Gegensatz der früheren Beweisführungen und der gegenwärtigen aus der Vertheilung und Natur der Farben an pleochromatischen Krystallen liegt vorzüglich darin, dal's jene sich auf einen einzigen polarisirten Lichtstrahl inwerbalb seiner Polarisationsebene in seiner Lage gegen die Trennungsfläche zweier verschieden dichter durchsichtiger Mittel beziehen, hier aber der Polarisationszustand nicht nur einer ganzen Ebene, sondern sogar der Polarisationszustand dreier senkrecht auf einander stehender Polarisationsebenen in allen Azimuten an jedem einzelnen Krystalle Gegenstand der Beobachtung und der auf sie zu gründenden Schlüsse ist. 